\title{
ON HOMOLOGY OF VIRTUAL BRAIDS AND BURAU REPRESENTATION
}

\author{
VLADIMIR V. VERSHININ
}

\begin{abstract}
Virtual knots arise in the study of Gauss diagrams and Vassiliev invariants of usual knots. The group of virtual braids on $n$ strings $V B_{n}$ and its Burau representation to $G L_{n} \mathbb{Z}\left[t, t^{-1}\right]$ also can be considered. The homological properties of the series of groups $V B_{n}$ and its Burau representation are studied. The following splitting of infinite loop spaces is proved for the plus-construction of the classifying space of the virtual braid group on the infinite number of strings:
\end{abstract}

$$
\mathbb{Z} \times B V B_{\infty}^{+} \simeq \Omega^{\infty} S^{\infty} \times S^{1} \times Y,
$$

where $Y$ is an infinite loop space. Connections with $K_{*} \mathbb{Z}$ are discussed.

\section{Introduction}

Virtual knots were introduced recently by L. Kauffman $[\mathrm{K}]$ and studied also by M. Goussarov, M. Polyak and O. Viro [GPV]. The main motivation lies in the theory of Gauss diagrams and Gauss codes of knots [K, PV]. Namely, for any knot diagram it is possible to construct its Gauss diagram and form its Gauss code. The example of a Gauss diagram of a knot is given on the Figure 1.

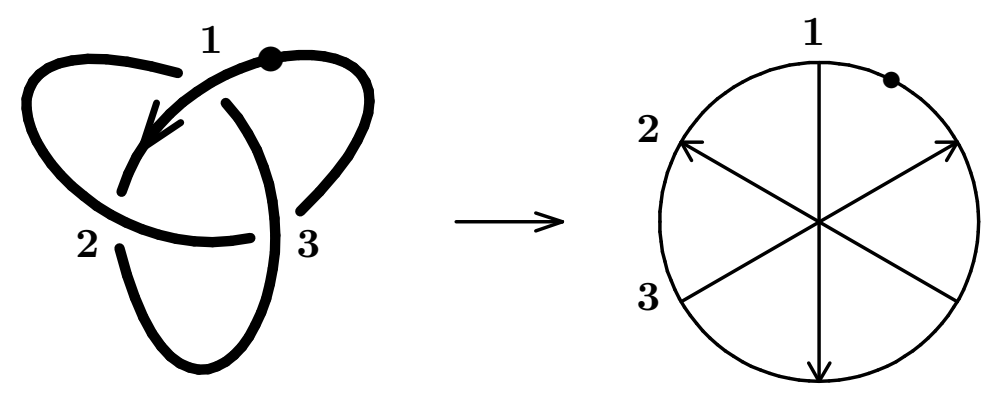

Fig. 1

The Gauss code of this Gauss diagram is the following:

O1U2O3U1O2U3,

where figures mean the crossing points and the symbol "O" means overcrossing and the symbol "U" means undercrossing at this point. The problem is that not every

1991 Mathematics Subject Classification. Primary 20J05, 20F36, 20F38, 18D10, 55P35.

Key words and phrases. Braid group, permutation group, classifying space, loop space.

This research was supported in part by INTAS Grant 96-0712. 
Gauss diagram (or Gauss code) corresponds to some knot. To escape this difficulty virtual knots were introduced. Really virtual knots are those whose Gauss diagram is not a Gauss diagram of any proper knot. L. Kauffman generalized many notions from the classical knot theory to virtual knots, such as fundamental group, rack, quandle, Kauffman and Jones polynomials. M. Goussarov, M. Polyak and O. Viro [GPV] proved that the analogues of the upper and the lower presentations of the classical fundamental group of a knot give two different groups for virtual knots. They also investigated virtual knots from the point of view of Vassiliev invariants.

Virtual braids correspond naturally to virtual knots. Some other generalizations of braids were introduced during the last several years. These are the braidpermutation group $B P_{n}$ of R. Fenn, R. Rimányi and C. Rourke $[\mathrm{FRR}]$ and the Baez-Birman monoid $S B_{n}$ [Ba, Bi2] which embeds into the singular braid group $S G_{n}[\mathrm{FKR}]$ (see also the description of these objects in the section 3 of the present papaer). Virtual braids are connected closely with both of them. They have the generators of the same types and the relations for virtual braids are of one type of relations for the braid-permutation group, which do not belong to the Baez-Birman monoid. All these three types of groups contain the classical braid groups. Burau representation is defined naturally for the virtual braids and the braid-permutation group, generalizing the classical Burau representation [Bu, Bi1].

Cohomologies of classical braid groups appeared in the papers of V. I. Arnold [Arn1, Arn2]. Their study is connected with various mathematical disciplines and questions. The classical Burau representation was considered from homotopical point of view by F. Cohen [C]. Cohomologies of the singular braid group $S G_{n}$ and of the braid-permutation group were studied by the author [Ve]. In the present paper we apply the methods of [ $\mathrm{Ve}]$ to the investigation of homological properties of the virtual braid group and its Burau representation.

\section{Virtual Braid Group}

The following analogues of Reidemeister moves were considered for virtual knots $[\mathrm{K}, \mathrm{GPV}]$ :
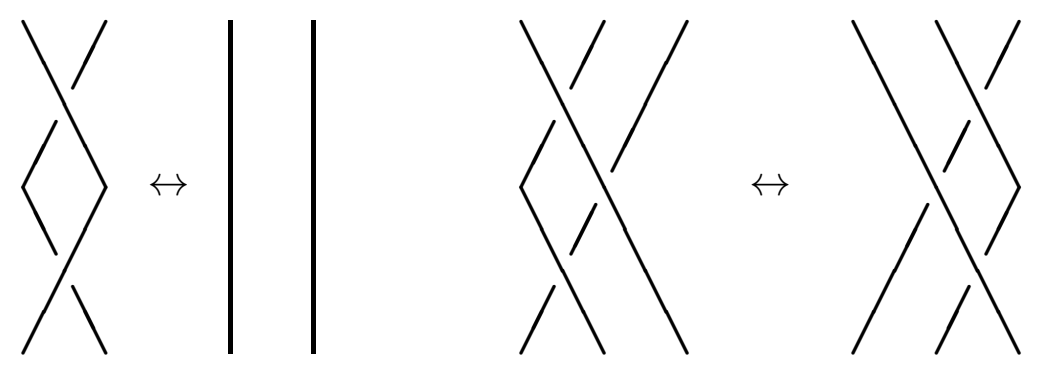

Fig. 2 


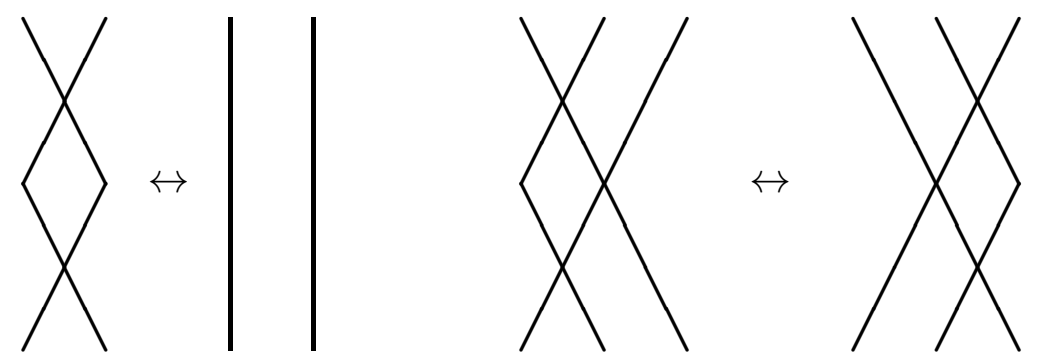

Fig. 3

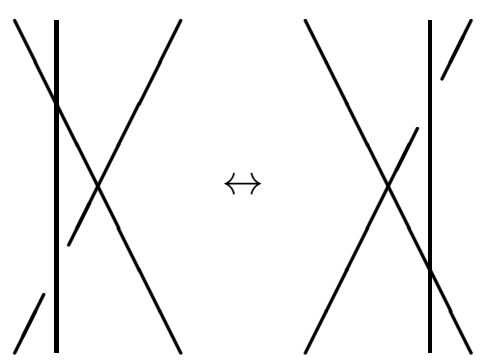

Fig. 4

It was emphasized in [GPV] that the following relation does not fulfill for virtual knots.

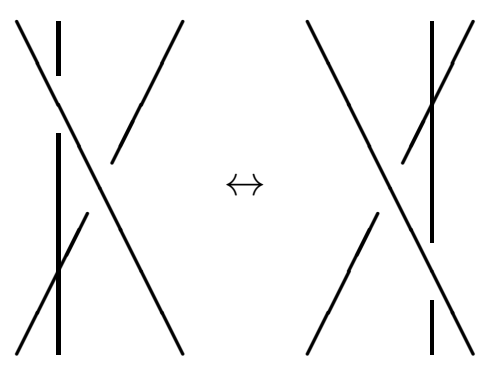

Fig. 5. Forbidden move

We can introduce the virtual braid group by the analogy with the classical braid group. The difference is that the two types of crossings are allowed: 1) as usual braids, what is shown at the Figure 6, or 2) as an intersection of lines on the plane as depicted at the Figure 7.
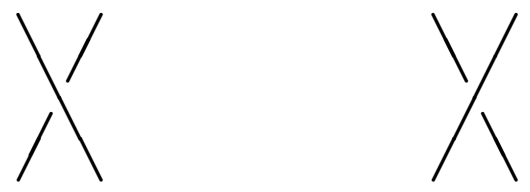

Fig. 6 


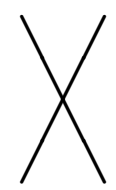

Fig. 7

This group is given by the following set of generators: $\left\{\zeta_{i}, \sigma_{i}, i=1,2, \ldots, n-1\right\}$ and relations:

$$
\begin{cases}\zeta_{i}^{2} & =1 \\ \zeta_{i} \zeta_{j} & =\zeta_{j} \zeta_{i}, \quad \text { if }|i-j|>1 \\ \zeta_{i} \zeta_{i+1} \zeta_{i} & =\zeta_{i+1} \zeta_{i} \zeta_{i+1}\end{cases}
$$

The symmetric group relations

$$
\begin{cases}\sigma_{i} \sigma_{j} & =\sigma_{j} \sigma_{i}, \text { if }|i-j|>1 \\ \sigma_{i} \sigma_{i+1} \sigma_{i} & =\sigma_{i+1} \sigma_{i} \sigma_{i+1}\end{cases}
$$

The braid group relations

$$
\begin{cases}\sigma_{i} \zeta_{j} & =\zeta_{j} \sigma_{i}, \text { if }|i-j|>1 \\ \zeta_{i} \zeta_{i+1} \sigma_{i} & =\sigma_{i+1} \zeta_{i} \zeta_{i+1}\end{cases}
$$

The mixed relations

The generator $\sigma_{i}$ corresponds to the canonical generator of the braid group $B r_{n}$ and is depicted at the Figure 8 .

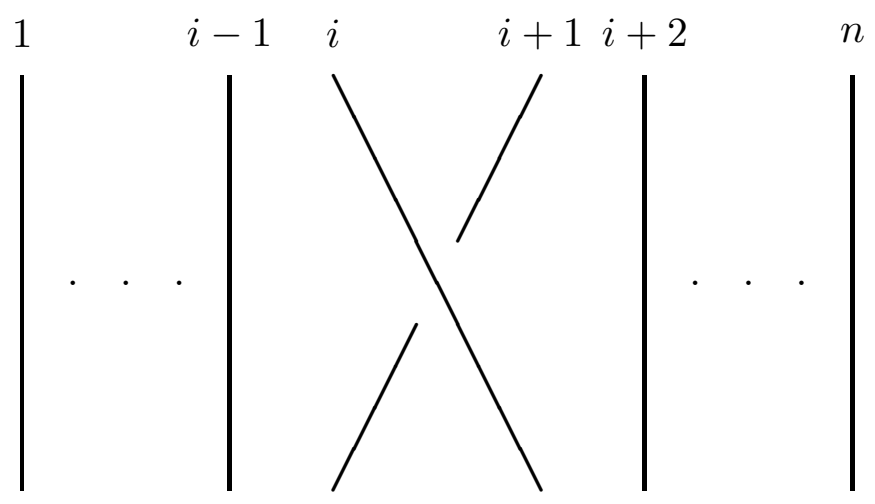

Fig. 8

The generators $\zeta_{i}$ correspond to the intersection of lines and is depicted at the Figure 9. 


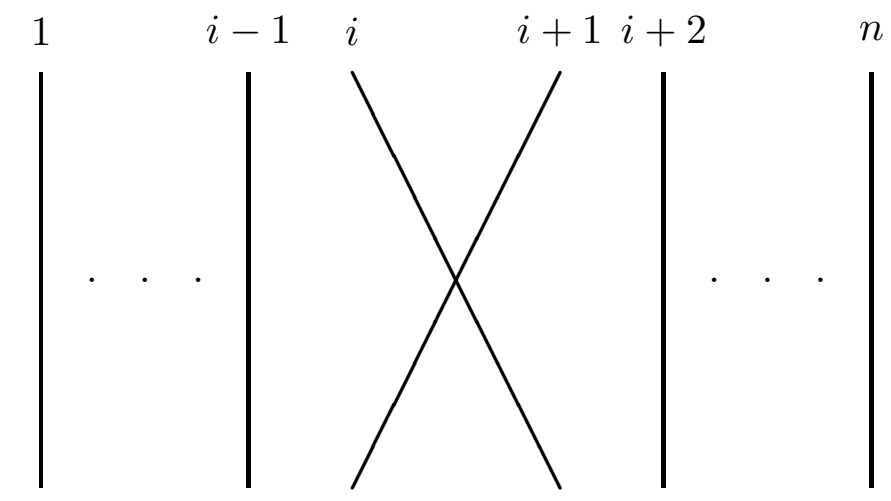

Fig. 9

A homomorphism $j_{n}$ from the classical braid group $B r_{n}$

$$
j_{n}: B r_{n} \rightarrow V B_{n}
$$

is evidently defined by the formulas

$$
j_{n}\left(\sigma_{i}\right)=\sigma_{i}
$$

We define the category $\mathcal{V B}$ by analogy with the case of the classical braids. Its objects $\{\overline{0}, \overline{1}, \ldots\}$ correspond to integer numbers from 0 to infinity and morphisms are defined by the formula:

$$
\operatorname{hom}(\bar{k}, \bar{l})= \begin{cases}V B_{k}, & \text { if } \quad k=l \\ \emptyset, & \text { if } k \neq l\end{cases}
$$

The pairings

$$
\mu_{m, n}: V B_{m} \times V B_{n} \rightarrow V B_{m+n}
$$

are defined in a usual way by the formulas

$$
\begin{gathered}
\mu_{m, n}\left(\sigma_{i}^{\prime}\right)=\sigma_{i}, \mu_{m, n}\left(\zeta_{i}^{\prime}\right)=\zeta_{i} ; \\
\sigma_{i}^{\prime}, \zeta_{i}^{\prime} \in V B_{m} ; \sigma_{i}, \zeta_{i} \in V B_{m+n} ; \\
\mu_{m, n}\left(\sigma_{j}^{\prime \prime}\right)=\sigma_{j+m}, \mu_{m, n}\left(\zeta_{j}^{\prime \prime}\right)=\zeta_{j+m} ; \\
\sigma_{i}^{\prime \prime}, \zeta_{i}^{\prime \prime} \in V B_{n} ; \sigma_{j+m}, \zeta_{j+m} \in V B_{m+n} .
\end{gathered}
$$

Geometrically this pairing is defined the same way as for the classical braid groups: we add (for example on the right) a braid with $n$ strings to a braid with $m$ strings. It is evident that the pairings for virtual braids agree with the corresponding pairings for the classical braid groups. They define a strict monoidal category $\mathcal{V B}$ and homomorphisms $j_{n}$ define the functor from the strict monoidal category $\mathcal{B}$ generated by the braid groups:

$$
\mathcal{J}: \mathcal{B} \rightarrow \mathcal{V B}
$$

which is a morphism of monoidal categories.

We remind that the category $\mathcal{B}$ is a braided category as defined by A. Joyal and R. Street [JS]. This means that there is given a system of isomorphisms of objects

$$
\sigma_{\bar{m}, \bar{n}}: \overline{m+n} \rightarrow \overline{n+m}
$$


natural with respect to morphisms from $\bar{m}$ to itself and from $\bar{n}$ to itself and satisfying the properties of coherence B1 and B2 from [JS]. A braided category becomes symmetric monoidal category if its braiding satisfies the additional property:

$$
\sigma_{\bar{m}, \bar{n}} \sigma_{\bar{n}, \bar{m}}=1 \overline{m+n} \text {. }
$$

We consider the following system of elements as a braiding $c$ in $\mathcal{B}$

$$
\sigma_{m} \ldots \sigma_{1} \sigma_{m+1} \ldots \sigma_{2} \ldots \sigma_{n+m-1} \ldots \sigma_{n} \in B r_{m+n}
$$

Graphically it is depicted at the Figure 10.

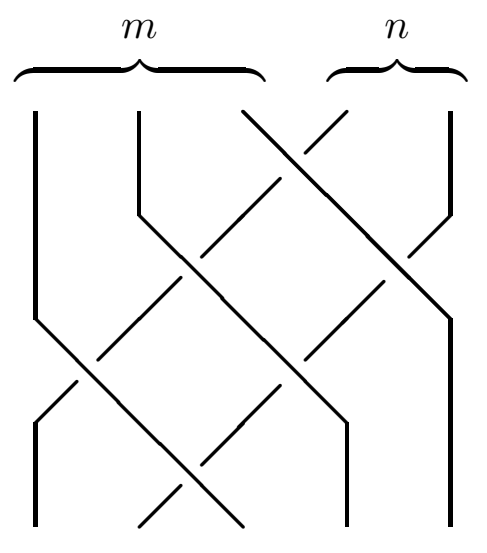

Fig. 10

Because of the forbidden move (Fig. 5) it does not follow that the image of the braiding $c$ in the category $\mathcal{B}$ by the functor $\mathcal{J}$ defines a braiding in the category $\mathcal{V B}$.

\section{The Braid-Permutation Group and the Baez-Birman Monoid}

Let $F_{n}$ be the free group of rank $n$ with the set of generators $\left\{x_{1}, \ldots, x_{n}\right\}$ and Aut $F_{n}$ be the automorphisms group of $F_{n}$. There are the standard inclusions of the symmetric group $\Sigma_{n}$ and the braid group $B r_{n}$ into Aut $F_{n}$. They can be described in the following way. Let $\xi_{i} \in$ Aut $F_{n}, i=1,2, \ldots, n-1$, be given by the formula of the action on the generators:

$$
\begin{cases}x_{i} & \mapsto x_{i+1} \\ x_{i+1} & \mapsto x_{i} \\ x_{j} & \mapsto x_{j}, j \neq i, i+1\end{cases}
$$

Let $\sigma_{i} \in$ Aut $F_{n}, i=1,2, \ldots, n-1$, be given by the formula of the action on the on generators:

$$
\begin{cases}x_{i} & \mapsto x_{i+1} \\ x_{i+1} & \mapsto x_{i+1}^{-1} x_{i} x_{i+1} \\ x_{j} & \mapsto x_{j}, j \neq i, i+1\end{cases}
$$

If we map the standard generators of the symmetric group to $\xi_{i}$ and the standard generators of the braid group to $\sigma_{i}$, then we get the monomorphisms $t_{\Sigma}$ and $t_{B}$ of groups:

$$
t_{\Sigma}: \Sigma_{n} \rightarrow \text { Aut } F_{n}
$$




$$
t_{B}: B r_{n} \rightarrow \text { Aut } F_{n}
$$

Let $B P_{n}$ be the subgroup of Aut $F_{n}$, generated by the both sets of automorphisms $\xi_{i}$ and $\sigma_{i}$ of (2) and (3). It is called the braid-permutation group. R. Fenn, R. Rimányi and C. Rourke proved [FRR] that this group is given by the following set of generators: $\left\{\xi_{i}, \sigma_{i}, i=1,2, \ldots, n-1\right\}$ and relations: the braid group relations for the generators $\sigma_{i}$ (the same as in (1)), and also:

$$
\begin{cases}\xi_{i}^{2} & =1 \\ \xi_{i} \xi_{j} & =\xi_{j} \xi_{i}, \text { if }|i-j|>1 \\ \xi_{i} \xi_{i+1} \xi_{i} & =\xi_{i+1} \xi_{i} \xi_{i+1} .\end{cases}
$$

The symmetric group relations

$$
\begin{cases}\sigma_{i} \xi_{j} & =\xi_{j} \sigma_{i}, \text { if }|i-j|>1, \\ \xi_{i} \xi_{i+1} \sigma_{i} & =\sigma_{i+1} \xi_{i} \xi_{i+1}, \\ \sigma_{i} \sigma_{i+1} \xi_{i} & =\xi_{i+1} \sigma_{i} \sigma_{i+1} .\end{cases}
$$

The mixed relations

R. Fenn, R. Rimányi and C. Rourke gave the geometrical interpretation of $B P_{n}$ as a group of welded braids. At first they define a welded braid diagram on $n$ strings as a collection of $n$ monotone arcs starting from $n$ points at a horizontal line of a plane (the top of the diagram) and going down to $n$ points at another horizontal line (the bottom of the diagram). It is allowed for them to have crossings of two types: 1) as usual braids, what is shown at the Figure 6, or 2) to have welds as depicted at the Figure 11.

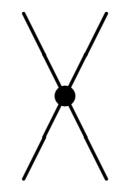

Fig. 11

An example of a welded braid diagram is shown at the Figure 12.

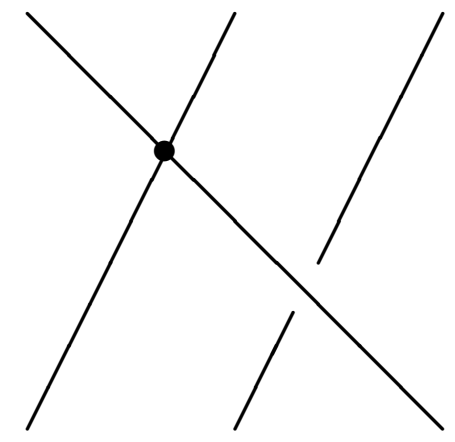

Fig. 12

The composition of welded braid diagrams on $n$ strings is defined by stacking. The diagram with no crossings or welds is an identity with respect to this composi- 
tion. So the set of welded braid diagrams on $n$ strings forms a semi-group denoted by $W D_{n}$.

R. Fenn, R. Rimányi and C. Rourke define the following types of allowable moves on welded braid diagrams. They are depicted at the Figures 13-16.
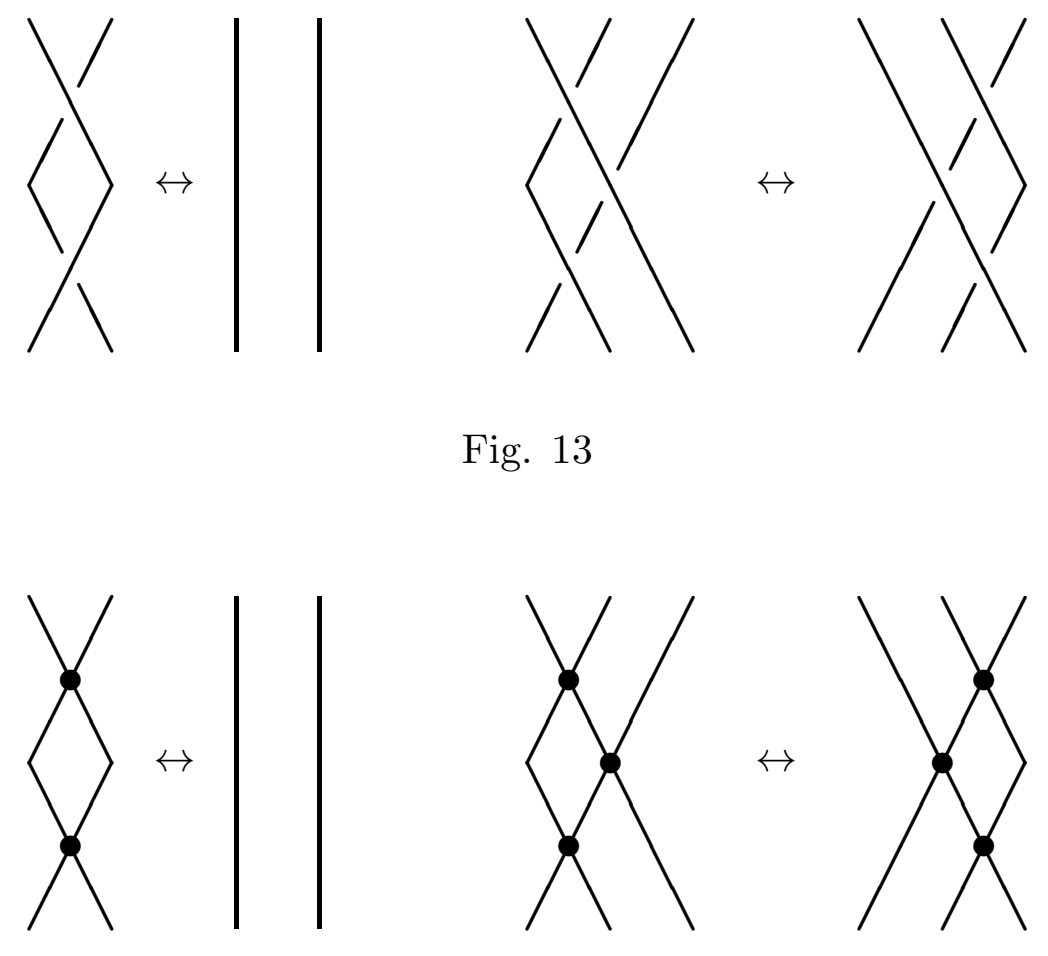

Fig. 14
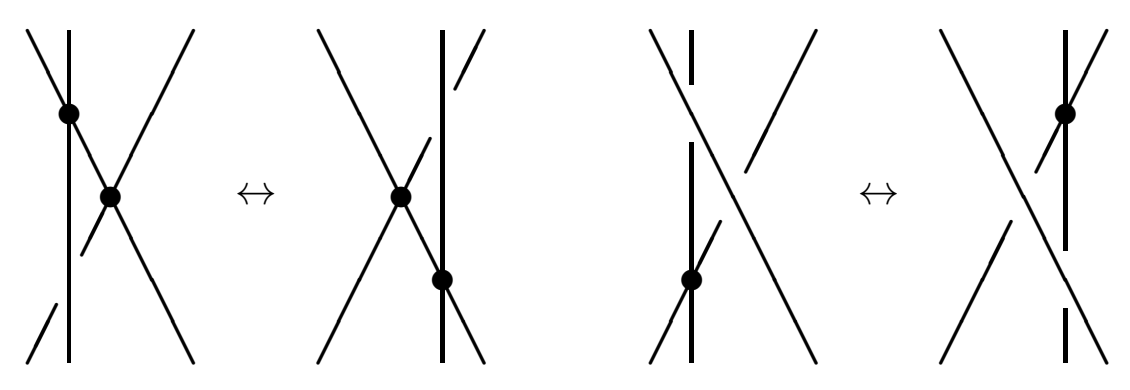

Fig. 15

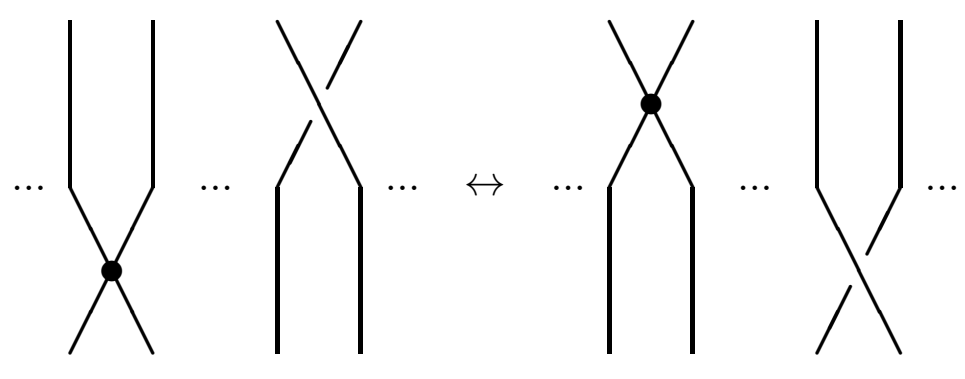

Fig. 16 


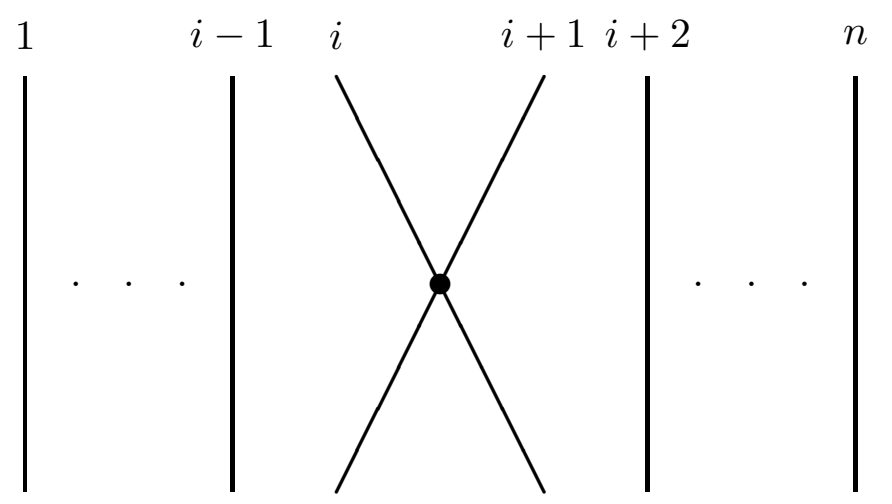

Fig. 17

The move from the Figure 16 is the geometric form of the commutativity from the mixed relations. There are also analogous moves corresponding to the commutativity from the symmetric group and the braid group relations.

A welded braid is defined as an equivalence class of welded braid diagrams under allowable moves. R. Fenn, R. Rimányi and C. Rourke proved that welded braids form a group and this group is isomorphic to the braid-permutation group $B P_{n}$. The generator $\sigma_{i}$ corresponds to the canonical generator of the braid group $B r_{n}$ and is depicted at the Figure 8. The generators $\xi_{i}$ correspond to the welded braids depicted at the Figure 17.

It is also possible to consider welded braids as objects of a 3-dimensional space. We regard them as imbedded in the positive half (with respect to the third coordinate) of a 3-dimensional space, while the welds are supposed to belong to the two-dimensional plane with the third coordinate equal to zero. Hence strings are not allowed to move behind welds.

The Baez-Birman monoid $S B_{n}$ (or generalized braid monoid, or singular braid monoid) $[\mathrm{Ba}, \mathrm{Bi} 2]$ is defined as a monoid with generators $\sigma_{i}, \sigma_{i}^{-1}, a_{i}, i=1, \ldots, n-1$, and relations

$$
\begin{aligned}
& \sigma_{i} \sigma_{j}=\sigma_{j} \sigma_{i}, \text { if }|i-j|>1, \\
& a_{i} a_{j}=a_{j} a_{i}, \text { if }|i-j|>1, \\
& a_{i} \sigma_{j}=\sigma_{j} a_{i}, \text { if }|i-j| \neq 1, \\
& \sigma_{i} \sigma_{i+1} \sigma_{i}=\sigma_{i+1} \sigma_{i} \sigma_{i+1}, \\
& \sigma_{i} \sigma_{i+1} a_{i}=a_{i+1} \sigma_{i} \sigma_{i+1}, \\
& \sigma_{i+1} \sigma_{i} a_{i+1}=a_{i} \sigma_{i+1} \sigma_{i}, \\
& \sigma_{i} \sigma_{i}^{-1}=\sigma_{i}^{-1} \sigma_{i}=1 .
\end{aligned}
$$

In pictures $\sigma_{i}$ corresponds to canonical generator of the braid group (right-handed crossing) and $a_{i}$ represents an intersection of the $i$ th and $(i+1)$ st strand, just as in Figures 8 and 17. More detailed geometric interpretation of the Baez-Birman monoid can be found in the paper of J. Birman [Bi2].

A homomorphism $k_{n}$ from the braid group $B r_{n}$ is evidently defined:

$$
k_{n}: B r_{n} \rightarrow S B_{n}
$$

R. Fenn, E. Keyman and C. Rourke proved in [FKR] that the Baez-Birman monoid embeds in a group $S G_{n}$ which they call the singular braid group:

$$
S B_{n} \rightarrow S G_{n} .
$$


It means that the elements $a_{i}$ become invertible and all the relations of $S B_{n}$ remain true.

We see that in the two types of mixed relations of the braid-permutation group one comes from the virtual braid group and another one comes from the BaezBirman monoid. Comparing presentations of the virtual braid group and the braidpermutation group the evident epimorphism can be defined by the formulas:

$$
\begin{gathered}
p\left(\zeta_{i}\right)=\xi_{i}, \\
p\left(\sigma_{i}\right)=\sigma_{i} .
\end{gathered}
$$

Considering the braid-permutation group as a subgroup of the automorphism group of the free group Aut $F_{n}$ we see that the homomorphism $p$ defines a representation of the virtual braid group in Aut $F_{n}$.

\section{Burau Representation}

Let us map the generators of the braid-permutation group $B P_{n}$ to the following elements of the group $G L_{n} \mathbb{Z}\left[t, t^{-1}\right]$

$$
\begin{gathered}
\sigma_{i} \mapsto\left(\begin{array}{cccc}
E_{i-1} & 0 & 0 & 0 \\
0 & 1-t & t & 0 \\
0 & 1 & 0 & 0 \\
0 & 0 & 0 & E_{n-i-1}
\end{array}\right), \\
\xi_{i} \mapsto\left(\begin{array}{cccc}
E_{i-1} & 0 & 0 & 0 \\
0 & 0 & 1 & 0 \\
0 & 1 & 0 & 0 \\
0 & 0 & 0 & E_{n-i-1}
\end{array}\right) .
\end{gathered}
$$

Proposition 1. The formulas (4) and (5) define correctly the representation of the braid-permutation group in $G L_{n} \mathbb{Z}\left[t, t^{-1}\right]$ :

$$
r: B P_{n} \rightarrow G L_{n} \mathbb{Z}\left[t, t^{-1}\right]
$$

Proof. If follows from the Fox free differential calculus or can be checked directly.

We denote by $B u P_{n}$ the image in $G L_{n} \mathbb{Z}\left[t, t^{-1}\right]$ of the braid-permutation group, which is also the image of the virtual braid group. So we have the homomorphisms:

$$
V B_{n} \stackrel{p}{\longrightarrow} B P_{n} \stackrel{r}{\longrightarrow} B u P_{n}
$$

We need the following simple fact about the group $V B_{n}$.

Proposition 2. The abelianizations of $V P_{n}$ and $B u P_{n}, 2 \leq n \leq \infty$, are both equal to $\mathbb{Z} / 2 \oplus \mathbb{Z}$ :

$$
\begin{gathered}
B V_{n} /\left[V B_{n}, V B_{n}\right] \cong \mathbb{Z} / 2 \oplus \mathbb{Z}, \\
B u P_{n} /\left[B u P_{n}, B u P_{n}\right] \cong \mathbb{Z} / 2 \oplus \mathbb{Z} .
\end{gathered}
$$


The homomorphisms $p$ and $r$ become isomorphisms after the abelianization.

Proof. We follow the lines of the proof of the analogous fact for the braid-permutation group [Ve]. Let us add new relations to that of the virtual braid group $V B_{n}$ :

$$
\begin{aligned}
\zeta_{i} & =\zeta_{j} \text { for all } i \text { and } j, \\
\sigma_{i} & =\sigma_{j} \text { for all } i \text { and } j,
\end{aligned}
$$

and obtain the epimorphism

$$
a b_{V B}: V B_{n} \rightarrow \mathbb{Z} / 2 \oplus \mathbb{Z} .
$$

On the other hand if we consider the free product of the symmetric and the braid groups $\Sigma_{n} * B r_{n}$, we obtain from the universality that the homomorphism of abelianization for the group $\Sigma_{n} * B r_{n}$ can be defined as the composition

$$
a b_{\Sigma_{n} * B r_{n}}: \Sigma_{n} * B r_{n} \stackrel{a b_{\Sigma * a b_{B r}}}{\longrightarrow} \mathbb{Z} / 2 * \mathbb{Z} \rightarrow \mathbb{Z} / 2 \oplus \mathbb{Z},
$$

where the second map is the canonical epimorphism. Consider the homomorphism $a b_{\Sigma * B r_{n}}$ as the composition

$$
\Sigma_{n} * B r_{n} \rightarrow V B_{n} \stackrel{a b_{V \beta}}{\longrightarrow} \mathbb{Z} / 2 \oplus \mathbb{Z},
$$

where the first map is the canonical epimorphism. Again using the universality we see that $a b_{V B}$ is the abelianization of $V B_{n}$. For the group $V B_{n}$ the assertion is proved. The determinant of the matrix in (5) is equal to $-t$ and the determinant of the matrix in (4) is equal to -1 . So we have an epimorphism to $\mathbb{Z} / 2 \oplus \mathbb{Z}$. The rest of the proof follows from the first part.

There is an epimorphism

$$
\alpha_{n}: V B_{n} \rightarrow \mathbb{Z},
$$

which is given by the formulas

$$
\begin{aligned}
\zeta_{i} & \mapsto 0 \text { for all } i, \\
\sigma_{i} & \mapsto 1 \text { for all } i .
\end{aligned}
$$

It follows from the relations, that there exists the epimorphism

$$
\phi_{n}: V B_{n} \rightarrow \Sigma_{n},
$$

defined by the formulas:

$$
\begin{aligned}
\phi_{n}\left(\zeta_{i}\right) & =\zeta_{i}, \\
\phi_{n}\left(\sigma_{i}\right) & =\zeta_{i} .
\end{aligned}
$$

Its composition with the canonical inclusion $\nu_{n}$ of $\Sigma_{n}$ in $V B_{n}$ is equal to the identity map of $\Sigma_{n}$. These homomorphisms generate maps of classifying spaces $B \nu_{n}$ and $B \phi_{n}$, such that their composition

$$
B \Sigma_{n} \rightarrow B V B_{n} \rightarrow B \Sigma_{n}
$$


is equal to identity. We have also the inclusion $j_{n}$ of the braid group in $V B_{n}$, which generates the map of classifying spaces:

$$
B B r_{n} \rightarrow B B V_{n}
$$

The composition of $j_{n}$ and $\phi_{n}$ gives the canonical epimorphism:

$$
\tau_{n}: B r_{n} \rightarrow \Sigma_{n}
$$

We denote by $\mathcal{Z}$ a strict monoidal (tensor) category whose objects $\{\overline{0}, \overline{1}, \ldots\}$ correspond to integer numbers from 0 to infinity and morphisms are defined by the formula:

$$
\operatorname{hom}(\bar{k}, \bar{l})= \begin{cases}\mathbb{Z}, & \text { if } \quad k=l, \\ \emptyset, & \text { if } \quad k \neq l .\end{cases}
$$

The product in $\mathcal{Z}$ is defined on objects by the sum of nonnegative numbers and on morphisms by the sum of integer numbers. This category has a symmetry which is equal to zero element for all $\bar{m}$ and $\bar{n}$. The homomorphisms $\alpha_{n}$ induce a morphism of permutative categories

$$
A: \mathcal{V B} \rightarrow \mathcal{Z}
$$

and maps of classifying spaces

$$
B \alpha_{n}: B V B_{n} \rightarrow S^{1}
$$

We denote by $\gamma$ an inclusion of the group $\mathbb{Z}$ into $B r_{n}$ :

$$
\gamma: \mathbb{Z} \rightarrow B r_{n}
$$

when the generator of the cyclic group is mapped to one of the generators $\sigma_{i}$, say, $\sigma_{1}: \gamma(1)=\sigma_{1}$.

Theorem 1. There exist maps

$$
\psi_{V}: B \Sigma \times B B r \rightarrow B V B^{+}
$$

and

$$
\beta_{V}: \Omega B\left(\amalg_{n \geq 0} B V B_{n}\right) \rightarrow \Omega^{\infty} S^{\infty} \times S^{1},
$$

such that the map $\psi_{V}$ becomes a loop map after the group completion, the map $\beta_{V}$ is an infinite loop map and it splits by the map

$$
\mathbb{Z} \times B \Sigma^{+} \times S^{1} \stackrel{I d \times(B \gamma)^{+}}{\longrightarrow} \mathbb{Z} \times B \Sigma^{+} \times B B r^{+} \stackrel{I d \times \psi_{V}^{+}}{\longrightarrow} \mathbb{Z} \times B V B^{+} .
$$

If an infinite loop space $Y$ is a fibre of the map $\beta_{V}$, then it fits for the following splitting of the infinite loop spaces:

$$
\Omega B\left(\amalg_{n \geq 0} B V B_{n}\right) \simeq \Omega^{\infty} S^{\infty} \times S^{1} \times Y .
$$

The same is true for the groups $B u P_{n}$ : the exist the maps $\psi_{P}$ and $\beta_{P}$ with analogous properties and they commute with the maps $\psi_{V}$ and $\beta_{V}$ and corresponding maps for the braid-permutation group with the help of maps induced by $p$ and $r$.

Proof. We follow the lines of work [Ve]. The category $\mathcal{V B}$ becomes a permutative category with the symmetry defined by the elements

$$
\zeta_{\bar{m}, \bar{n}}=\zeta_{m} \ldots \zeta_{1} \zeta_{m+1} \ldots \zeta_{2} \ldots \zeta_{n+m-1} \ldots \zeta_{n} \in V B_{m+n}
$$


It is evident that

$$
\zeta^{2}=1
$$

By definition, the naturality of the symmetry $\zeta$ means that the following equality

$$
\zeta \bar{m}, \bar{n} \cdot \mu\left(b_{m}^{\prime}, b_{n}^{\prime \prime}\right)=\mu\left(b_{n}^{\prime \prime}, b_{m}^{\prime}\right) \cdot \zeta \bar{m}, \bar{n}, \quad b_{m}^{\prime} \in V B_{m}, \quad b_{n}^{\prime \prime} \in V B_{n}
$$

is fulfilled. This is equivalent to the expression

$$
\zeta \bar{m}, \bar{n} \cdot \mu\left(b_{m}^{\prime}, b_{n}^{\prime \prime}\right) \cdot \zeta \bar{m}, \bar{n}=\mu\left(b_{n}^{\prime \prime}, b_{m}^{\prime}\right)
$$

which means that the conjugation by the element $\zeta \bar{m}, \bar{n}$ transforms the elements of $V B_{m} \times V B_{n}$, canonically lying in $V B_{m+n}$, into the corresponding elements of $V B_{n} \times V B_{m}$. The elements $\zeta \bar{m}, \bar{n}$ define a symmetry for the category formed by symmetric groups, so, for checking the naturality of $\zeta$ in $\mathcal{V B}$ it remains to verify the naturality for the generators $\sigma_{i}, 1 \leq i \leq m-1, m \leq i \leq m+n$. Let us consider the corresponding conjugation:

$$
\zeta_{m} \ldots \zeta_{1} \zeta_{m+1} \ldots \zeta_{2} \ldots \zeta_{n+m-1} \ldots \zeta_{n} \sigma_{i} \zeta_{n} \ldots \zeta_{n+m-1} \ldots \zeta_{2} \zeta_{m+1} \zeta_{1} \ldots \zeta_{m}
$$

If $i>n$, we use the relation

$$
\zeta_{i-1} \sigma_{i} \zeta_{i-1}=\zeta_{i} \sigma_{i-1} \zeta_{i}
$$

We have:

$$
\begin{gathered}
\zeta_{m} \ldots \zeta_{1} \zeta_{m+1} \ldots \zeta_{2} \ldots \zeta_{n+m-1} \ldots \zeta_{n} \sigma_{i} \zeta_{n} \ldots \zeta_{n+m-1} \ldots \zeta_{2} \zeta_{m+1} \zeta_{1} \ldots \zeta_{m}= \\
\zeta_{m} \ldots \zeta_{1} \zeta_{m+1} \ldots \zeta_{2} \ldots \zeta_{n+m-1} \ldots \zeta_{i-1} \sigma_{i} \zeta_{i-1} \ldots \zeta_{n+m-1} \ldots \zeta_{2} \zeta_{m+1} \zeta_{1} \ldots \zeta_{m}= \\
\zeta_{m} \ldots \zeta_{1} \zeta_{m+1} \ldots \zeta_{2} \ldots \zeta_{n+m-1} \ldots \zeta_{i+1} \sigma_{i-1} \zeta_{i} \zeta_{i-1} \zeta_{i-1} \ldots \zeta_{n+m-1} \ldots \zeta_{2} \zeta_{m+1} \zeta_{1} \ldots \zeta_{m}= \\
\ldots=\sigma_{i-n} .
\end{gathered}
$$

If $i<n$, we use the relation

$$
\zeta_{i} \sigma_{i+1} \zeta_{i}=\zeta_{i+} \sigma_{i} \zeta_{i+1}
$$

We have:

$$
\begin{gathered}
\zeta_{m} \ldots \zeta_{1} \zeta_{m+1} \ldots \zeta_{2} \ldots \zeta_{n+m-1} \ldots \zeta_{n} \sigma_{i} \zeta_{n} \ldots \zeta_{n+m-1} \ldots \zeta_{2} \ldots \zeta_{m+1} \zeta_{1} \ldots \zeta_{m}= \\
\zeta_{m} \ldots \zeta_{1} \zeta_{m+1} \ldots \zeta_{2} \ldots \zeta_{i} \zeta_{i+m} \ldots \zeta_{i+2} \zeta_{i+1} \sigma_{i} \zeta_{i+1} \ldots \zeta_{i+m} \ldots \zeta_{2} \zeta_{m+1} \zeta_{1} \ldots \zeta_{m}= \\
\zeta_{m} \ldots \zeta_{1} \zeta_{m+1} \ldots \zeta_{2} \ldots \zeta_{i} \zeta_{i+m} \ldots \zeta_{i+2} \zeta_{i} \sigma_{i+1} \zeta_{i} \zeta_{i+2} \ldots \zeta_{i+m} \ldots \zeta_{1} \ldots \zeta_{m}= \\
\zeta_{m} \ldots \zeta_{1} \zeta_{m+1} \ldots \zeta_{2} \ldots \zeta_{i+1} \zeta_{i+m} \ldots \zeta_{i+2} \sigma_{i+1} \zeta_{i+2} \ldots \zeta_{i+m} \zeta_{i+1} \ldots \zeta_{2} \ldots \zeta_{m+1} \zeta_{1} \ldots \zeta_{m}=\ldots= \\
=\sigma_{i+m} .
\end{gathered}
$$

The conditions of coherence are fulfilled trivially. For the condition B1 of [JS] we have identically:

$$
\zeta_{m} \ldots \zeta_{1} \zeta_{m+1} \ldots \zeta_{2} \ldots \zeta_{n+m-1} \ldots \zeta_{n} \cdot \zeta_{m+n} \ldots \zeta_{n+1} \zeta_{m+n+1} \ldots \zeta_{n+2} \ldots \zeta_{n+m+q-1} \ldots \zeta_{n+q}=
$$




$$
=\zeta_{m} \ldots \zeta_{1} \zeta_{m+1} \ldots \zeta_{2} \ldots \zeta_{n+m+q-1} \ldots \zeta_{n+q} .
$$

For B2 it is also evident:

$$
\begin{gathered}
\zeta_{m+n} \ldots \zeta_{m+1} \zeta_{m+n+1} \ldots \zeta_{m+2} \ldots \zeta_{n+m+q-1} \ldots \zeta_{m+q} \cdot \zeta_{m} \ldots \zeta_{1} \zeta_{m+1} \ldots \zeta_{2} \ldots \zeta_{m+q-1} \ldots \zeta_{q}= \\
=\zeta_{m+n} \ldots \zeta_{1} \zeta_{m+n+1} \ldots \zeta_{2} \ldots \zeta_{m+n+q-1} \ldots \zeta_{q}
\end{gathered}
$$

So, we proved that the category $\mathcal{V B}$ is a permutative category. The morphism $A$ is a morphism of permutative categories and hence induces the map of infinite loop spaces

$$
\Omega B\left(\amalg_{n \geq 0} B V B_{n}\right) \rightarrow S^{1} .
$$

Analogously the homomorphisms $\phi_{n}$ generate the morphism of the permutative categories

$$
\mathcal{V B} \rightarrow \Sigma
$$

and the corresponding infinite loop map

$$
\Omega B\left(\amalg_{n \geq 0} B V B_{n}\right) \rightarrow \Omega^{\infty} S^{\infty} .
$$

We denote by $\beta_{V}$ the following composition:

$$
\Omega B\left(\amalg_{n \geq 0} B V B_{n}\right) \stackrel{\text { diag }}{\longrightarrow} \Omega B\left(\amalg_{n \geq 0} B V B_{n}\right) \times \Omega B\left(\amalg_{n \geq 0} B V B_{n}\right) \rightarrow \Omega^{\infty} S^{\infty} \times S^{1} .
$$

The homomorphisms $\nu$ and $j$ induce the map of products of classifying spaces:

$$
B \Sigma \times B B r \rightarrow B V B \times B V B .
$$

The space $B V B^{+}$is an $H$-space, so there exists a map

$$
\mu: B V B^{+} \times B V B^{+} \rightarrow B V B^{+}
$$

Let us consider now the composition $f$ :

$$
B \Sigma \times B B r \stackrel{B \nu \times B j}{\longrightarrow} B V B \times B V B \stackrel{q \times q}{\longrightarrow} B V B^{+} \times B V B^{+} \stackrel{\mu}{\longrightarrow} B V B^{+},
$$

where $q$ is the canonical map to the plus-construction

$$
q: B V B \rightarrow B V B^{+}
$$

From the construction we see that the map

$$
B \Sigma \stackrel{\text { equiv }}{\longrightarrow} B \Sigma \times * \stackrel{i d \times i n c l}{\longrightarrow} B \Sigma \times B B r \stackrel{f}{\longrightarrow} B V B^{+}
$$

is homotopic to $q B \nu$, and the map

$$
B B r \stackrel{\text { equiv }}{\longrightarrow} * \times B B r \stackrel{\text { incl } \times i d}{\longrightarrow} B \Sigma \times B B r \stackrel{f}{\rightarrow} B V B^{+}
$$

is homotopic to $q B j$. Using the fact that $\mathbb{Z} \times B V B^{+}$is a loop space, we change a little the map

$$
q B j: B B r \rightarrow B V B^{+}
$$


and define the map

$$
g: B B r \rightarrow B V B^{+}
$$

by the formula

$$
g(b)=(q B \tau(b))^{-1} \cdot q B j(b), b \in B B r .
$$

In this case the composition

$$
B^{+} \phi \cdot g: B B r \rightarrow B \Sigma^{+}
$$

is homotopic to zero. We denote by $\psi$ the following composition

$$
B \Sigma \times B B r \stackrel{B \nu \times g}{\longrightarrow} B V B \times B V B \stackrel{q \times q}{\longrightarrow} B V B^{+} \times B V B^{+} \stackrel{\mu}{\longrightarrow} B V B^{+}
$$

and by $\chi$ the composition

$$
B \Sigma \times S^{1} \stackrel{i d \times B \gamma}{\longrightarrow} B \Sigma \times B B r \stackrel{\psi}{\longrightarrow} B V B^{+} .
$$

So, the composition

$$
B \Sigma \times B B r \stackrel{\psi}{\longrightarrow} B V B^{+} \stackrel{B^{+}}{\longrightarrow \times B^{+} \alpha} B \Sigma^{+} \times S^{1}
$$

is homotopic to the product $q \times B \alpha$ and the composition

$$
B \Sigma \times S^{1} \stackrel{\chi}{\longrightarrow} B V B^{+} \stackrel{B^{+} \phi \times B^{+} \alpha}{\longrightarrow} B \Sigma^{+} \times S^{1}
$$

is the canonical map from a space to its plus-construction. It gives the following splitting:

$$
B \Sigma^{+} \times S^{1} \stackrel{\chi^{+}}{\longrightarrow} B V B^{+^{+}} \stackrel{B^{+} \phi \times B^{+} \alpha}{\longrightarrow} B \Sigma^{+} \times S^{1} .
$$

The proof for the groups $B u P_{n}$ is the same. We notice only that $K_{1} \mathbb{Z}\left[t, t^{-1}\right]$ is isomorphic to the group of units of the ring $\mathbb{Z}\left[t, t^{-1}\right]$ which is isomorphic to $\mathbb{Z} / 2 \oplus \mathbb{Z}$ :

$$
\mathbb{Z}\left[t, t^{-1}\right]^{*} \cong \mathbb{Z} / 2 \oplus \mathbb{Z}
$$

and the map is given by determinant:

$$
G L\left(\mathbb{Z}\left[t, t^{-1}\right]\right) \stackrel{\text { det }}{\longrightarrow} \mathbb{Z}\left[t, t^{-1}\right]^{*} .
$$

It follows from the Proposition 1 that Burau representation defines a map

$$
B V B^{+} \rightarrow B G L\left(\mathbb{Z}\left[t, t^{-1}\right]\right)^{+} .
$$

Let us consider the homomorphism of homotopy groups that it induces.

$$
\pi_{*}\left(B V B^{+}\right) \rightarrow \pi_{*}\left(B G L\left(\mathbb{Z}\left[t, t^{-1}\right]\right)^{+} .\right.
$$

On the left hand side of (6) we have $\pi_{*}\left(\Omega^{\infty} S^{\infty} \times S^{1} \times Y\right) \cong \pi_{*}^{S} \oplus \pi_{*}\left(S^{1}\right) \oplus \pi_{*}(Y)$, where $\pi_{*}^{S}$ are the stable homotopy groups of spheres, and on the right hand side $K_{*} \mathbb{Z}\left[t, t^{-1}\right] \cong K_{*} \mathbb{Z} \oplus K_{*-1} \mathbb{Z}$. 
Proposition 3. The map (6) on the level of $\pi_{1}$ generates an epimorphism, which is an isomorphism on the direct summand:

$$
\pi_{1}\left(\Omega^{\infty} S^{\infty} \times S^{1}\right) \stackrel{\simeq}{\longrightarrow} K_{1} \mathbb{Z} \oplus K_{0} \mathbb{Z} \cong \mathbb{Z} / 2 \oplus \mathbb{Z}
$$

For the summand $\pi_{*}\left(\Omega^{\infty} S^{\infty}\right)$ the map (6) factors through $K_{*} \mathbb{Z}$ where it is equal to the canonical homomorphism

$$
\pi_{*}^{S} \rightarrow K_{*} \mathbb{Z}
$$

The proof follows directly from constructions.

The map (7) as well as the groups $K_{*} \mathbb{Z}$ were studied in algebraic K-theory (see, for example [Q, We]). The ranks of the groups $K_{*} \mathbb{Z}$ were calculated by A. Borel [Bo]. Results of V. Voevodsky [Vo] allowed to C. Weibel [W] (with some clarifications of J. Rognes and C. Weibel $[\mathrm{RW}]$ ) to complete the determination of the 2-torsion in the groups $K_{*} \mathbb{Z}$.

Initially D. Quillen $[\mathrm{Q}]$ considered the commutative diagram

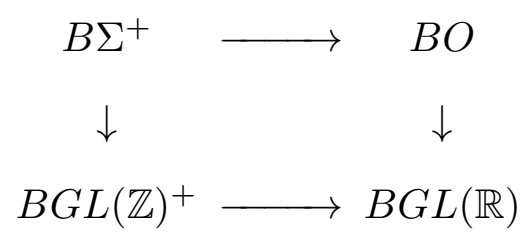

and used the results of J. F. Adams on J-homomorphism [Ad]. When $i$ is $8 k+1$ or $8 k+2$, the 2 -torsion part of $K_{i} \mathbb{Z}$ is equal to $\mathbb{Z} / 2$ and is generated by the images of Adams' elements $\mu_{i} \in \pi_{*}^{S}$. When $i=8 k+3$ the 2 -subgroup of the image of J-homomorphism is $\mathbb{Z} / 8$ and is contained in the 2 -subgroup of $K_{i} \mathbb{Z}$ equal to $\mathbb{Z} / 16$. When $i=8 k+7$ the 2 -subgroup of the image of J-homomorphism is equal to $\mathbb{Z} / w_{j}$ and is isomorphic to the 2 -subgroup of $K_{i} \mathbb{Z}$, where $j=4(k+1)$ and $w_{j}$ is the largest power of 2 dividing $4 j$.

\section{Acknowledgements}

The author is thankful to Misha Polyak, who explained him the work [GPV] and to John Rognes for useful information. He is also grateful to Sofia Lambropoulou and all the organizers of the conference Knots-98 in Delphi, Greece for the work that they carried out in connection with this very interesting and successful meeting.

\section{REFERENCES}

[Ad] J. F. Adams, On the groups $J(X)$. IV., Topology 5 (1966), 21-71.

[Ar1] V. I. Arnold, On some topological invariants of algebraic functions, Trudy Moscov. Mat. Obshch. 21 (1970), 27-46 (Russian), English transl. in Trans. Moscow Math. Soc. 21 (1970), 30-52.

[Ar2] V. I. Arnold, Topological invariants of algebraic functions II, Funct. Anal. Appl. 4, No 2 (1970), 1-9 (Russian),.

[Ba] J. C. Baez, Link Invariants of Finite Type and Perturbation Theory, Lett. Math. Physics 26 (1992), 43-51.

[Bi1] J. S. Birman, Braids, Links, and Mapping Class Groups, Ann. Math. Stud., No 82, 1974.

[Bi2] J. S. Birman, New points of view in knot theory, Bull. Amer. Math. Soc. 28, No 2 (1993), $253-387$.

[Bo] A. Borel, Cohomologie réelle stable de groupes S-arithmétiques classiques, C. R. Acad. Sci. Paris Sér. A-B 274 (1972), A1700-A1702. 
[Bu] W. Burau, Über Zopfgruppen und gleichsinnig verdrillte Verkettunger, Abh. Math. Semin. Hanischen Univ. 11 (1936), 171-178.

[C] F. Cohen, Artin's braid groups and classical homotopy theory, Contemp. Math. 44, 1985, 207-220.

[FKR] R. Fenn, E. Keyman and C. Rourke, The Singular Braid Monoid Embeds in a Group, Preprint, 1996.

[FRR] R. Fenn, R. Rimányi and C. Rourke, The Braid-Permutation Group, Topology 36, No 1 (1997).

[GPV] M. Goussarov, M. Polyak, O. Viro, Finite Type Invariants of Classical and Virtual Knots, Preprint. October 1998. http://xxx.lanl.gov/math.GT/9810073.

[JS] A. Joyal and R. Street, Braided tensor categories, Adv. Math. 102 (1993), 20-78.

[K] L. Kauffman, Virtual Knot theory, Preprint. 1998. http://xxx.lanl.gov/math.GT/9811028,

[PV] M. Polyak and O. Viro, Gauss Diagram Formulas for Vassiliev Invariants, Internat. Math. Res. Notices No. 11 (1994), 445-453.

[Q] D. Quillen, Letter from Quillen to Milnor on $\operatorname{Im}\left(\pi_{i} 0 \rightarrow \pi_{i}^{\mathrm{s}} \rightarrow K_{i} Z\right)$, Algebraic $K$-theory (Proc. Conf., Northwestern Univ., Evanston, Ill., 1976), Lecture Notes in Math., Vol. 551, Springer, Berlin, 1976, 182-188.

[RW] J. Rognes and C. Weibel, Two-Primary Algebraic K-Theory of Rings of Integers in Number Fields, Preprint.

[Ve] V. V. Vershinin, On Homological Properties of Singular Braids, Trans. Amer. Math. Soc. 350, No 6 (1998), 2431-2455.

[Vo] V. Voevodsky, The Milnor Conjecture, Preprint MPIM. 1996.

[We] C. Weibel, The 2-torsion in the K-theory of the Integers, C. R. Acad. Sci. 324, Série I (1997), 615-620.

Sobolev Institute of Mathematics, Novosibirsk, 630090, Russia

E-mail address: versh@math.nsc.ru 